\title{
Digging into the 3D Quantum Hall Effect
}

\author{
Theorists invoke electron-phonon interactions to explain the recent \\ observation of the quantum Hall effect in a 3D electronic system.
}

\author{
By Luis E. F. Foa Torres
}

n February 1980, Klaus von Klitzing made a discovery that opened up one of the most exciting chapters in physics history. He had prepared a semiconductor device containing electrons confined to a single layer. This so-called 2D electron gas was already well known to physicists. But when von Klitzing subjected it to very low temperature and very high magnetic field, he found that an intrinsic electronic property, the Hall

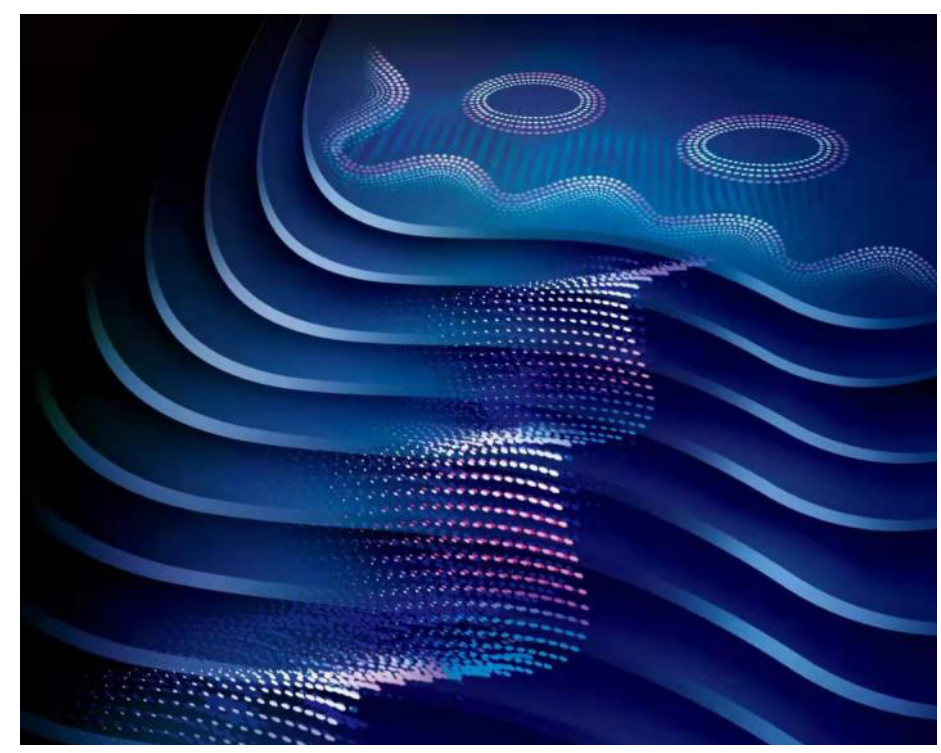

Figure 1: The usual quantum Hall effect emerges in a sheet of electrons that is pierced with a strong magnetic field. The phenomenon is quintessentially $2 \mathrm{D}$, but it can emerge in a $3 \mathrm{D}$ system if the system's electrons form a charge-density wave (CDW) along the magnetic-field direction. As shown here, the CDW is a standing wave of electrons along the vertical direction.

Credit: Wang Guoyan \& He Cong resistance, occurred only at quantized values that were integer multiples of $h / e^{2}[1]$. The exceptional precision of those values, and their observed insensitivity to sample impurities, ultimately led to the quantum Hall resistance being used to redefine a unit in terms of fundamental constants (see Kilogram Untethered from Early Objects). A perhaps lesser known fact is that physicists have been pursuing a 3D version of the quantum Hall effect (QHE) for 30 years. Experiments achieved success in 2019 [2]. Now, theorists are explaining those results with a model that involves a wave-like electron density. Their picture could help expand the realm of the QHE in 3D [3].

Like a box of chocolates, the QHE comes in many flavors [4]. The behavior seen by von Klitzing is known as the integer QHE. Physicists have since discovered a fractional version, a version that doesn't require a magnetic field, and even a light-induced version. In all cases, the effect is fundamentally tied to the system's two dimensionality. The Hall resistance measures how easily charges moving in an applied electric field can be "bent" by a perpendicular magnetic field. This resistance, which depends on the occupancy of available electronic states, varies continuously with magnetic field in a typical material. But in a very cold $2 \mathrm{D}$ system in a strong enough magnetic field, the Hall resistance only rises in steps (plateaus) because the field forces electrons in the bulk of the material to lie in flat bands with a quantized energy (Landau levels). As long as the Fermi energy, which characterizes the maximum energy of the electron distribution, lies in the gap between the levels, the Hall resistance is stuck at a plateau.

The QHE is difficult to achieve in 3D because the Landau levels spread out in energy along the direction of the magnetic field. 
As a result, no matter where the Fermi energy lies, the Landau levels (or, more accurately, bands) contribute states, filling the gap and destroying the Hall-resistance plateaus. But in 1987, Bert Halperin proposed that these problems would go away if some intrinsic instability of the material's electronic structure opened a gap in its electronic structure [5]. This instability could, for example, be an induced lattice potential or it could be a charge-density wave (CDW), where electrons form a standing wave pattern instead of being evenly distributed throughout the solid (Fig. 1). This clustering leads to an energy gap, and Halperin predicted that two concrete effects would result from the Fermi energy lying within this gap [5]: the resistivity along the electric field (the longitudinal resistivity) would be dissipationless and drop to zero; and the Hall resistivity would be restricted to the value $\lambda h / e^{2}$. Here, $\lambda$ is set by the period of some internal potential along the direction of the magnetic field, such as the lattice itself or a CDW. These predictions are precise hallmarks for distinguishing the 3D QHE from its 2D counterpart.

Several groups have attempted to see the 3D QHE [6-9], but none observed Halperin's hallmarks. One experimental challenge is the need to find a material with the right kind of instability. Another is that tried-and-true tools for manipulating $2 \mathrm{D}$ systems, such as using an electronic "gate" to tune the carrier concentration and the Fermi level, don't necessarily carry over to 3D. Researchers have tried to build a 3D system from stacks of 2D materials [10]. But the resulting shape of the Fermi surface indicates that the system is still 2D in nature.

The 2019 experiment that succeeded in observing the 3D QHE was performed on the compound $\mathrm{ZrTe}_{5}$ by Fangdong Tang of the Southern University of Science and Technology (SUSTech), China, and colleagues [2]. The researchers used a magnetic field of around $2 \mathrm{~T}$ and cooled their material samples to $0.6 \mathrm{~K}$. Crucially, these samples had high electronic mobilities and the Fermi level could be tuned to the "extreme quantum limit," where only the lowest Landau level is occupied. Under these conditions, the experiments showed suppressed longitudinal resistivity and a Hall resistivity plateau that existed between 1.7 and 2.2 T. Moreover, $\lambda$ for the plateau was equal to half the Fermi wavelength along the magnetic-field direction for all samples. Since the Fermi wavelength in these experiments was much larger than the lattice constant along the field direction, the researchers concluded that the observed 3D QHE arose from some long-wavelength potential (like a CDW) and not from any crystal potential.

Physicists have puzzled over two points about these new results: the precise mechanism behind the observed 3D QHE and why the Hall plateau exists only within a certain magnetic-field range. The theory from Fang Qin, also of SUSTech, and colleagues addresses both problems [3]. In their model, a magnetically induced CDW is behind the 3D QHE. They find that unlike CDWs that are driven by an electron-electron interaction (which would require very strong magnetic fields), CDWs driven by electron-phonon interaction can explain the experiments. They show that this interaction can create the required instability at the relatively small field of $1.7 \mathrm{~T}$. Their model also predicts that fields above about $2.1 \mathrm{~T}$ "depin" the CDW, making it incommensurate with the crystalline lattice. This feature explains why no resistance plateaus are seen above 2.1 T. The theorists' picture thus shows the dual function of the magnetic field: it induces an order parameter (CDW) in one direction and a topological phase (the QHE) in the perpendicular direction.

Decades after the discovery of the QHE, the family of related phenomena continues to spread in new directions. Experiment and theory on the 3D QHE open many possibilities that researchers have yet to tap. As Qin and colleagues suggest [3], the race is on for prospective materials hosting the 3D effect. Finding such materials is a must for this area to bloom. With a suitable handcrafting of the crystal structure and of the electron interactions, researchers may achieve a 3D QHE effect under less stringent experimental conditions, such as at room temperature or perhaps even without an external magnetic field. Chances are that condensed-matter physicists' beloved chocolate box is going to offer up more treats.

Luis E. F. Foa Torres: Faculty of Physics and Mathematical Sciences, University of Chile, Santiago, Chile

\section{REFERENCES}

1. K. von Klitzing et al., "New method for high-accuracy determination of the fine-structure constant based on quantized Hall resistance," Phys. Rev, Lett. 45, 494 (1980).

2. F. Tang et al., "Three-dimensional quantum Hall effect and metal-insulator transition in ZrTe 5 ," Nature 569, 537 (2019).

3. F. Qin et al., "Theory for the charge-density-wave mechanism 
of 3D quantum Hall effect," Phys. Rev. Lett. 125, 206601

(2020).

4. K. von Klitzing et al., "40 years of the quantum Hall effect," Nat. Rev. Phys. 2, 397 (2020).

5. B. I. Halperin, "Possible states for a three-dimensional electron gas in a strong magnetic field," Jpn J. Appl. Phys. 26, 1913 (1987).

6. J. R. K. Cooper et al., "Quantized Hall effect and a new field-induced phase transition in the organic superconductor $(\text { TMTSF })_{2}(\text { PF })_{6}$," Phys. Rev. Lett. 63, 1984 (1989).

7. S. T. Hannahs et al., "Quantum Hall effect in a bulk crystal,"
Phys. Rev. Lett. 63, 1988 (1989).

8. S. Hill et al., "Bulk quantum Hall effect in $\eta-\mathrm{Mo}_{4} \mathrm{O}_{11}$," Phys. Rev. B 58, 10778 (1998).

9. H. Cao et al., "Quantized Hall effect and Shubnikov-de Haas oscillations in highly doped $\mathrm{Bi}_{2} \mathrm{Se}_{3}$ : Evidence for layered transport of bulk carriers," Phys. Rev. Lett. 108, 216803 (2012).

10. H. L. Störmer et al., "Quantization of the Hall effect in an anisotropic three-dimensional electronic system," Phys. Rev. Lett. 56, 85 (1986). 